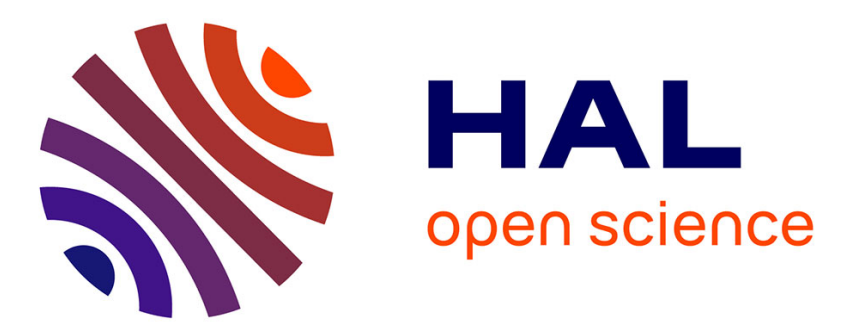

\title{
First Full-Dimensional Potential Energy and Dipole Moment Surfaces of SF6
}

\author{
Andrei V Nikitin, Michael M. Rey, Iana S Chizhmakova, Vladimir G Tyuterev
}

\section{To cite this version:}

Andrei V Nikitin, Michael M. Rey, Iana S Chizhmakova, Vladimir G Tyuterev. First Full-Dimensional Potential Energy and Dipole Moment Surfaces of SF6. Journal of Physical Chemistry A, 2020, 124 (35), pp.7014-7023. 10.1021/acs.jpca.0c02733 . hal-03045937

\section{HAL Id: hal-03045937 https://hal.science/hal-03045937}

Submitted on 8 Dec 2020

HAL is a multi-disciplinary open access archive for the deposit and dissemination of scientific research documents, whether they are published or not. The documents may come from teaching and research institutions in France or abroad, or from public or private research centers.
L'archive ouverte pluridisciplinaire HAL, est destinée au dépôt et à la diffusion de documents scientifiques de niveau recherche, publiés ou non, émanant des établissements d'enseignement et de recherche français ou étrangers, des laboratoires publics ou privés. 


\title{
FIRST FULL-DIMENSIONAL POTENTIAL ENERGY AND DIPOLE MOMENT SURFACES OF SF 6
}

\author{
Andrei V. Nikitin ${ }^{1}$, Michael Rey ${ }^{2}$, Iana S. Chizhmakova ${ }^{3,4}$, Vladimir G. Tyuterev ${ }^{2,4}$ \\ 1. V.E. Zuev Institute of Atmospheric Optics, Russian Academy of Sciences, 1, Akademichesky \\ Avenue, 634055 Tomsk, Russian Federation \\ 2. Groupe de Spectrométrie Moléculaire et Atmosphérique, UMR CNRS 6089, Université de Reims, \\ U.F.R. Sciences, B.P. 1039, 51687 Reims Cedex 2, France \\ 3. Institute of Monitoring of Climatic and Ecological Systems, Russian Academy of Sciences , 10/3, \\ Academichesky avenue, 634055, Tomsk, Russian Federation \\ 4. QUAMER laboratory, Tomsk State University, 36 Lenin Avenue, 634050 Tomsk, Russian \\ Federation
}

Running Head: potential energy surface and dipole moment surface of SF6

Keywords: SF6, ab initio, potential energy surface, dipole moment surface, low resolution spectra.

\section{Correspondence should be addressed to:}

Andrei V. Nikitin,

*Laboratory of Theoretical Spectroscopy, V.E. Zuev Institute of Atmospheric Optics, SB RAS, 1, Academician Zuev square, 634055, Tomsk, Russia

E-mail: avn@iao.ru

Phone : $+73822-491111$ (1260)

\section{ABSTRACT}


A 15-dimensional analytical form for the potential energy and dipole moment surfaces of the $\mathrm{SF}_{6}$ molecule in the ground electronic state is obtained using ab initio methods. In order to calculate the equilibrium S-F distance, we applied coupled cluster CCSD(T) method and several versions of the correlation consistent basis sets - from valence triple-zeta (VTZ) and augmented valence triple-zeta (AVTZ) to core-valence quadruple-zeta (CVQZ) with Douglas-Kroll (DK) relativistic corrections that provided good agreement with empirical equilibrium value. Ab initio electronic energies on 15D grids of nuclear geometries are computed using $\operatorname{CCSD}(\mathrm{T})$ method with VTZ and CVQZ-DK basis sets. The analytical representation of the potential energy surface is determined through an expansion in symmetry adapted products of non-linear coordinates up to the 5-th order. The influence of additional redundant coordinates on the quality of the fit was investigated. Parameters of full dimensional dipole moment surfaces are determined up to the 4th order expansion in normal mode coordinates. For validation of ab initio results, the fundamental vibration frequencies and absorption cross-sections of the principal sulfur hexafluoride isotopolgue are calculated giving good agreement with cold $(180 \mathrm{~K})$ and room temperature ( $296 \mathrm{~K}$ ) experimental spectra. Absorption cross-sections calculated from our preliminary line list agree much better with these observations than the simulations using $\mathrm{SF}_{6}$ line-by-line lists constructed from effective models included in currently available spectroscopic databases. octahedron

Keywords: Potential Energy Surface, Dipole moment surface, $\mathrm{SF}_{6}$, Octahedral Group

\section{Introduction}

Investigation of electronic structure and radiative properties of high octahedral symmetry sulfur hexafluoride $\left(\mathrm{SF}_{6}\right)$ molecule are of interest both for the theory $[\mathbf{1 , 2 , 3}$ and for applications in many domains of industry and medicine. Because of strong absorption bands in the atmospheric transparency windows, $\mathrm{SF}_{6}$ is a powerful greenhouse gas with the global warming potential (GWP500) by 29300 times larger than that of $\mathrm{CO}_{2}$ [4]. At present, the sulfur hexafluoride atmospheric concentration is yet small, but $\mathrm{SF}_{6}$ is one of the most stable gaseous chemicals with an atmospheric life time of 3200 years [5]. According to the conclusions of Earth System Research Laboratory Global Monitoring Division [6], the $\mathrm{SF}_{6}$ concentration increased from 3.5ppt (1995) to 9.5ppt (2018). A rapid increase of the $\mathrm{SF}_{6}$ concentration and its long atmospheric life time might result in a considerable accumulation of $\mathrm{SF}_{6}$ in the troposphere. Thus, in future, sulfur hexafluoride may play an important role in the climate global changing. The radiative properties of $\mathrm{SF}_{6}$ were insufficiently studied up to now. Because of a relatively low-frequency vibrations [7], the Boltzmann population of the excited vibrational levels appears to be significant. Thus, the "hot bands" corresponded to transitions between excited vibrational states produce a considerable contribution to IR absorption even at room temperature. The ro- 
vibrational spectra are quite complex and crowded making it difficult to identify isolated lines in experimentally recorded absorption samples. Only few vibrational bands of $\mathrm{SF}_{6}$ within limited spectral ranges have been studied [8,9] [10] [11] [12] using the effective Hamiltonian approach. Despite many efforts, a simulation of the room-temperature $\mathrm{SF}_{6}$ absorbance using effective spectroscopic models was not sufficiently complete. For this reason, the most advanced spectroscopic databases (as HITRAN [13] or GEISA [14] ) do not contain line parameters for all necessary $\mathrm{SF}_{6}$ bands in order to describe the absorbance at room temperature with high accuracy.

An approach based on ab initio potential energy surfaces (PES) and dipole moment surfaces (DMS) using variational methods for the solution of the full-dimensional nuclear motion Schrödinger equation, has been successfully applied to many 4-5 atomic [15] [16] [17] [18] [19] [20] [21] [22] [7], and also to six-atomic [23] [24] [25] molecules like $\mathrm{C}_{2} \mathrm{H}_{4}$. In [26] some calculated levels of $\mathrm{UF}_{6}$ molecule having the same octahedral symmetry have been reported. However, high precision full-dimensional PESs and DMSs for seven-atomic molecules are still lacking in the literature.

Several works used alternative methods for the nuclear motion calculations for medium and large molecules using vibrational configuration-interaction approach or the perturbation theory ( [27] [28] [29] and references herein). However in most cases the rotation states were computed for low $\mathbf{J}$ only and the contribution of hot bands have been neglected.

To compute energy levels and IR spectra beyond the harmonic oscillator approximation using $a b$ initio techniques, it is necessary to obtain either the PES on a sufficiently large grid of nuclear displacements or the force field with high order derivatives. Both techniques are often used, but for high-resolution applications the technique based on an analytical PES determined at an extended geometrical grid has proved to produce more accurate results [21] [30] [26]. In the full-grid approach, the computational difficulties are related to a simultaneous account of relatively large number of electrons (70 electrons in case of $\mathrm{SF}_{6}$ ) and of large number of degrees of freedom including representative nuclear geometries for the PES and DMS parametrization. As a rule, a number of different geometries is several times bigger than the number of the fitted PES parameters. The force field technique often uses the finite difference method for calculating the force constants, and as a rule, a validity of the obtained force field could not be checked for sufficiently large nuclear displacements. To reduce the number of nuclear geometries in the electronic structure calculations, it is necessary to take into account the molecular symmetry.

This paper is devoted to first calculations of the full-dimensional PES and DMS of $\mathrm{SF}_{6}$ molecule and is structured as follows. Equilibrium structure at various basis sets, the coordinate choice and analytical model for PES and DMS surfaces are considered in Section 2 whereas $a b$ initio calculations and fits are discussed in Sections 3 and 4. Calculation of the vibrational- 
rotation spectra using the variation techniques [31] [32] [33] [34] shows a good agreement with experimental absorption cross-sections in the range of the strongest bands of ${ }^{32} \mathrm{SF}_{6}$ and ${ }^{34} \mathrm{SF}_{6}$ isotopologues ( section 5), providing a preliminary validation of ab initio results.

\section{The equilibrium geometry and analytical form of PES and DMS}

The $\mathrm{SF}_{6}$ molecule belongs to the octahedral $O_{h}$ point group: at the equilibrium geometry, the molecule is a cube with the $\mathrm{S}$ atom at the cube center, and six $\mathrm{F}$ atoms at centers of each edge (Fig. 1). Thus, only one geometrical parameter (S-F distance) determines this equilibrium structure unambiguously. Experimental and theoretical studies of the $\mathrm{SF}_{6}$ equilibrium geometry have been considered in $[\mathbf{3 5}, 36]$. In order to calculate the equilibrium S-F distance, we applied coupled cluster $\operatorname{CCSD}(\mathrm{T})$ method and several versions of the correlation consistent basis sets from valence triple-zeta (VTZ) and augmented valence triple-zeta (AVTZ) to core-valence quadruple-zeta (CVQZ) with Douglas-Kroll (DK) relativistic corrections. All ab initio calculation were carried out using MOLPRO suite of programs as implemented in the 2019.1 version [37] [38]. More details concerning ab initio methods will be given in Section 3. A comparison of results obtained for various basis sets with some empirical values is collected in Table 1.

Table 1. Energies and the equilibrium lengths of the $\mathrm{S}-\mathrm{F}$ bound obtained with the optimization $\operatorname{CCSD}(\mathrm{T})$ technique using various basis sets.

$\begin{array}{lcc}\text { Method / basis set } & \text { Energy, Hartree } & \text { Equilibrium length S }-\mathrm{F}, \AA \\ \text { CCSD(T) / VTZ } & -996.07574167 & 1.57015425 \\ \text { CCSD }(\mathrm{T}) \text { / CVTZ } & -996.79947177 & 1.56003654 \\ \text { CCSD(T) / AVTZ } & -996.14253699 & 1.57357635 \\ \text { CCSD (T) / CVTZ - DK } & -998.23964874 & 1.56042314 \\ \text { CCSD }(\mathrm{T}) \text { / VQZ } & -996.30894816 & 1.56375885 \\ \text { CCSD (T) / CVQZ } & -997.10132168 & 1.55654004 \\ \text { CCSD (T) / CVQZ-DK } & -998.70581919 & 1.55702740 \\ \text { Empirical variational * } & & 1.556 \\ \text { Ref. [39] } & & 1.5647 \\ \text { Empirical value [40] } & & 1.5622\end{array}$

*Preliminary result of empirical optimization of $S-F$ distance using variational calculations of rotation energies [32] [7]

Two calculations with two basis sets CVQZ and CVQZ-DK give values closed to the empirical ones. In any case without additional corrections it is impossible to calculate the equilibrium geometry with a precision better than $0.001 \mathrm{~A}^{\circ}$. 


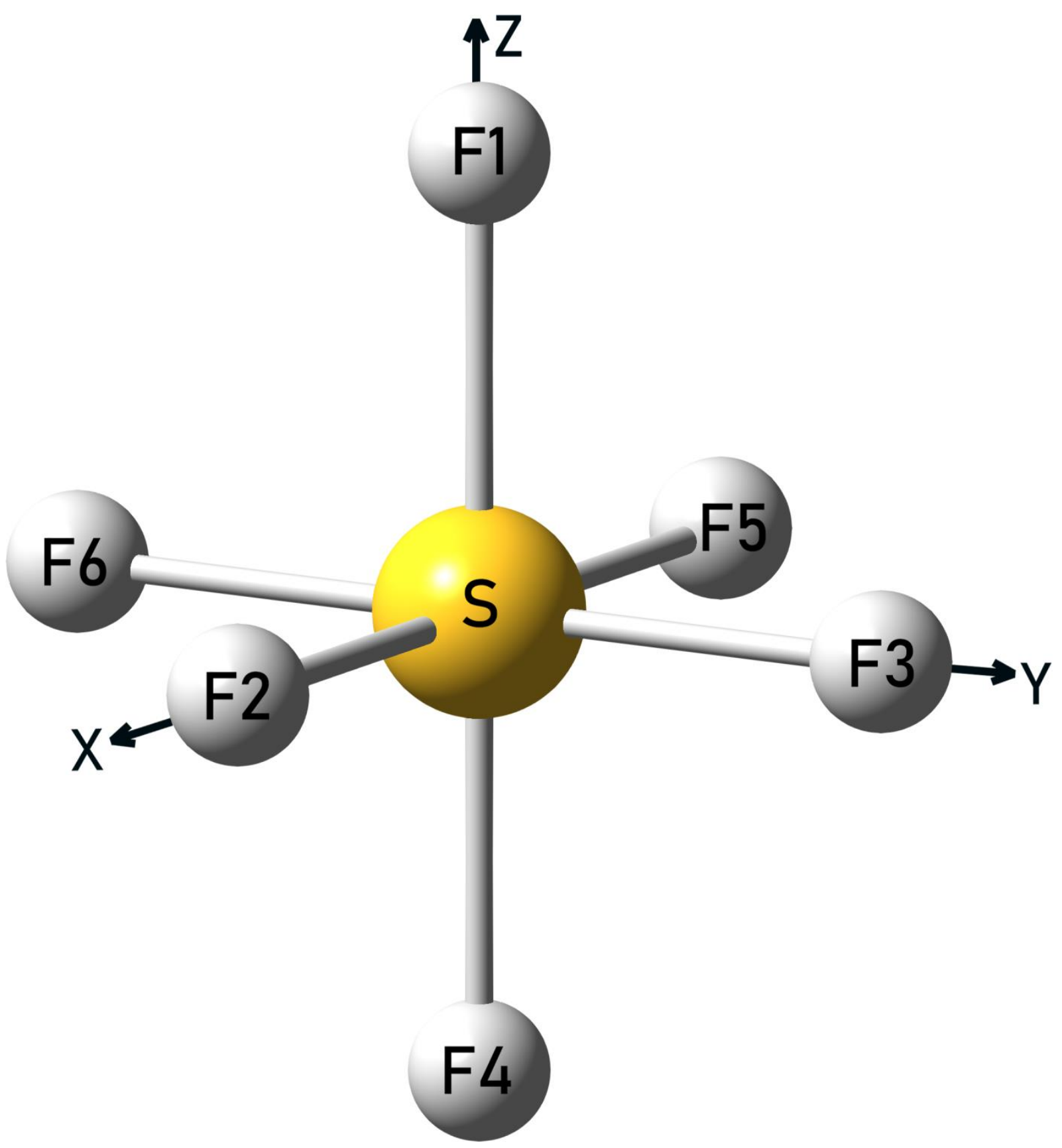

Figure 1. Equilibrium structure and the axes choice for the $\mathrm{SF}_{6}$ molecule

Various sets of the symmetry coordinates have been used in many previous works. Most of them are incompatible with our definitions of Clebsch-Gordan coefficients for the $O_{h}$ group [41] [42]. Here we use the symmetric coordinates and the molecule orientation from the work [43]. Initially, we begin with linear combinations of bond lengths and bond angles, which will be further adapted using non-linear elementary morse-type and cosine-type functions as described in Section 3. At the first step, six "radial" (stretching) symmetrized coordinates were used to accommodate three irreducible representations: $A_{1 g}, E_{g}$, and $F_{1 u}$.

$$
S R_{A_{1 g}}=\left(r_{1}+r_{2}+r_{3}+r_{4}+r_{5}+r_{6}-6 r_{e}\right) / \sqrt{6}
$$




$$
\begin{aligned}
& S R_{E_{g}(a)}=\left(2 r_{1}+2 r_{4}-r_{2}-r_{3}-r_{5}-r_{6}\right) / \sqrt{12} \\
& S R_{F_{1 u}(x)}=\left(r_{2}-r_{5}\right) / \sqrt{2}
\end{aligned}
$$

Nine angular coordinates $q_{i j}$ were symmetrized according to three irreducible representations: $F_{1 u}, \quad F_{2 g} F_{2 u}$.

$$
\begin{aligned}
& S A_{F_{1 u}(x)}=\left(q_{12}+q_{23}+q_{24}+q_{26}-q_{15}-q_{35}-q_{45}-q_{56}\right) / \sqrt{8} \\
& S A_{F_{2 g}(x)}=\left(q_{13}+q_{46}-q_{16}-q_{34}\right) / 2 \\
& S A_{F_{2 u}(x)}=\left(q_{15}+q_{23}+q_{26}+q_{45}-q_{12}-q_{24}-q_{35}-q_{56}\right) / \sqrt{8}
\end{aligned}
$$

Some of these $q_{i j}$ angles in Fig 1 , which take $\pi / 2$ values at the equilibrium are called "adjacent" angles. Another ones, which take $\pi$ values at the equilibrium are called "opposition" angles. The number of possible angles is bigger than the number of symmetrized angular coordinates. So two types of additional symmetrized angular coordinates can be added: three coordinates $S^{\prime \prime}$ of symmetry $A_{l g}, E_{g}$ composed of "opposition" angles $q_{14}, q_{25}, q_{36}$ or three coordinates $S^{\prime}$ of the same symmetry $A_{l g}, E_{g}$ from 12 "adjacent" angles :

$$
\begin{gathered}
S_{E_{a}}^{\prime \prime}=\left(q_{25}+q_{36}-2 q_{14}\right) / \sqrt{6} \\
S_{E_{b}}^{\prime \prime}=\left(q_{36}-q_{25}\right) / \sqrt{6} \\
S_{A_{1 g}}^{\prime \prime}=\left(q_{25}+q_{36}+q_{14}-3 q_{e}^{\prime \prime}\right) / \sqrt{3} \\
S_{E_{a}}^{\prime}=\left(q_{12}+q_{15}+q_{34}+q_{46}+q_{13}+q_{16}+q_{24}+q_{45}-2 q_{23}-2 q_{35}-2 q_{56}-2 q_{26}\right) / \sqrt{24} \\
S_{E_{b}}^{\prime}=\left(q_{12}+q_{24}+q_{15}+q_{45}-q_{13}-q_{46}-q_{34}-q_{16}\right) / \sqrt{8} \\
S_{A_{1 g}}^{\prime}=\left(q_{12}+q_{15}+q_{34}+q_{46}+q_{13}+q_{16}+q_{24}+q_{45}+q_{23}+q_{35}+q_{56}+q_{26}-12 q_{e}^{\prime}\right) / \sqrt{12}
\end{gathered}
$$

Here $r_{e}$ is the equilibrium values for the S-F distance, $q{ }^{\prime \prime}$ and $q_{e}^{\prime}$ are equilibrium values for "adjacent" or "opposite" angles. The additional angular coordinates can significantly accommodate the fit of the shape of the surface near the bottom of the potential well that will affect the energy levels and wave functions. In the case of methane, this has been shown in the Refs [21].

The complete set of coordinates and the $O_{h}$ group together with the matrices of irreducible representations (irrep) were reported in Ref [43]. As explained in [43], our set of internal angular coordinated is different from that of refs [44], [45], [46]. The definition of the radial symmetrized coordinates (1-3) coincides with that of [47]. Matrix representation of group elements [43] (see Table 1 of Appendix 1 for matrices) coincides with that of ref. [48] as given in our Table 2. 
Table 2. $O_{h}$ : point group elements

\begin{tabular}{|l|c|c|c|}
\hline Group element & $\mathrm{C}_{31}^{+}$ & $\mathrm{C}_{4 \mathrm{z}}^{+}$ & Inversion \\
\hline Transformation matrix & $\left(\begin{array}{lll}0 & 0 & 1 \\
1 & 0 & 0 \\
0 & 1 & 0\end{array}\right)$ & $\left(\begin{array}{ccc}0 & -1 & 0 \\
1 & 0 & 0 \\
0 & 0 & 1\end{array}\right)$ & $\left(\begin{array}{ccc}-1 & 0 & 0 \\
0 & -1 & 0 \\
0 & 0 & -1\end{array}\right)$ \\
\hline $\begin{array}{c}\text { Equivalent permutations } \\
\text { of F atoms (1 to 6) }\end{array}$ & $\begin{array}{c}123456 \\
312645 \\
\text { or } \\
(132)(465)\end{array}$ & $\begin{array}{c}123456 \\
162435 \\
\text { or } \\
(2653)\end{array}$ & $\begin{array}{c}123456 \\
456123 \\
\text { or } \\
(14)(25)(26)\end{array}$ \\
\hline
\end{tabular}

An account of the symmetry and the use of the irreducible tensor representation permitted obtaining an optimal non-redundant grid of nuclear geometries. One grid adapted for the analytical PES development up to the 6-th order (6412 geometries) has been constructed in the work [43] using the geometry set generation technique already applied for other symmetric molecules [49] [50]. Here we use also extended grid of 78739 geometries that unambiguously define the PES development up to 8-th order constructed by the same technique. These two grids of geometries allowed us to carry out $a b$ initio electronic energy calculations for a wide range of nuclear displacements. Initially, the grids were constructed in symmetrized coordinates (1-6) but for $a b$ initio calculations both grids were recalculated in internal coordinates as in ref [43]: six vectors connecting the $\mathrm{S}$ atom with the $\mathrm{F}$ atoms; six lengths of the $\mathrm{S}-\mathrm{F}$ bonds $\left\{r_{1}, r_{2}, r_{3}, r_{4}, r_{5}, r_{6}\right\}$; five angles between the bonds $\left\{q_{12}, q_{13}, q_{14}, q_{15}, q_{16}\right\}$, and four torsion angles $\left\{t_{23}, t_{24}, t_{25}, t_{26}\right\}$ (see Fig. 1).

In the analytical expressions for PES it is recommended to use the symmetry coordinates that are linear combinations of the internal coordinates or of 1D elementary functions of these internal coordinates. A seven-atomic molecule has 15 vibrational degrees of freedom. The standard representation in terms of the irreducible tensor operators (ITO) [41] [42] was used to obtain analytical expressions for 15-dimensional (15D) PES and DMS developments. Three stretching vibrational modes $v_{1}$ ( non-degenerate at $\left.774.5 \mathrm{~cm}^{-1}\right), v_{2}\left(\right.$ two-fold degenerate at $643.3 \mathrm{~cm}^{-1}$ ) and $v_{3}$ ( three-fold degenerate at $948.1 \mathrm{~cm}^{-1}$ ) transform according to the $A_{1 g}, E_{g}$ and $F_{1 u}$ irreducible representations while three triply degenerate bending modes $v_{4}, v_{5}$, and $v_{6}$ transform under the $F_{1 u}\left(615.0 \mathrm{~cm}^{-1}\right), F_{2 g}\left(524.0 \mathrm{~cm}^{-1}\right)$ and $F_{2 u}\left(347.7 \mathrm{~cm}^{-1}\right)$ representations.

The analytic potential function is written in terms of the irreducible tensor operators:

$$
V(R)=\sum_{i, p} F_{i p} R_{i}^{p}\left(r_{1}, r_{2}, r_{3}, r_{4}, r_{5}, r_{6}, q_{12}, q_{13}, q_{14}, q_{15}, q_{16}, t_{23}, t_{24}, t_{25}, t_{26}\right)
$$

where each $\mathbf{R}_{\mathrm{i}}^{\mathrm{p}}$ tensor is obtained as an appropriate product of radial $S R_{\Gamma}$ and angular $S A_{\Gamma}$ nonlinear symmetrized coordinates: 
$\boldsymbol{R}_{i}^{p}=\left(\left(\left[S R_{A 1_{g}}\right]^{p_{1}} \times\left(\left[S R_{E_{g}}\right]^{p_{2}} \times\left[S R_{F 1_{u}}\right]^{p_{3}}\right)^{C_{23}}\right)^{C^{\prime}} \times\left(\left[S A_{F 1_{u}}\right]^{p_{4}} \times\left(\left[S A_{F 2_{g}}\right]^{p_{5}} \times\left[S A_{F 2_{u}}\right]^{p_{6}}\right)^{C_{56}}\right)^{C^{\prime \prime}}\right)^{C}$.

These non-linear radial $S R_{\Gamma}$ and angular $S A \Gamma$ non-linear symmetrized coordinates involved in our PES model $(9,10)$ were obtained by replacing the combinations $(1-6)$ by the morse-type and cosine-type elementary functions in a similar way as was described in our works for the methane molecule [21] [49] [51]. We have tried several choices for an optimal fit to ab initio points. The final expressions of elementary functions used in the present work are given in the next Section 3. Here $p_{j}$ components of $p=\left\{p_{1}, \ldots p_{6}\right\}$ correspond to powers of these coordinates and $p=p_{1}+$ $p_{2}+\cdots+p_{6}$ is the total power of the expansion term. The lower case index i corresponds to the set of symbols $C_{23}, C_{56}, C, C^{\prime}, C^{\prime \prime}$ in the summation (9) that run over nondegenerate $\left(A_{1 g}\right)$, doubly $\left(E_{g}\right)$ and triply degenerate $\left(F_{1 u}, F_{2 u}\right.$, and $\left.F_{2 g}\right)$ irreducible representations (irreps) of $O_{h}$ point group. The $C_{23}$ irrep results from the coupling of the symmetrized powers of the second and the third vibrational modes, whereas $C^{\prime}$ stands for the irrep corresponding to the coupling of the latter product with the symmetrized power of the first vibrational mode. In a similar manner the irrep $C_{56}$ corresponds to the coupling of the fifth and sixth modes coupled with the fourth mode via $C^{\prime \prime}$. For the PES expansion $C^{\prime}=C^{\prime}$, so that only the final symmetry allowed type $C$ of the $R_{i}^{p}$ tensors is $A_{l g}$. Very similar expansion can be used for the DMS, but in this case the final symmetry type $C$ of the $R_{i}^{p}$ tensors is $F_{1 u}$. Each combination of the symmetrized coordinate powers $p_{1}, \ldots, p_{6}$ and of the coupling irreps $C_{23}, C_{56}, C, C$ ', $C$ " form a "tree" [41] [52]. An ITO model for the PES or for the DMS expansion at a given total order $p$ corresponds to the summation over various symmetry allowed trees [52] with the $F_{i p}$ parameters fitted to ab initio values. If the « redundant » symmetrized coordinates of the type $S^{\prime}(8)$ or $S^{\prime \prime}(7)$ are included in the PES model [43], then two more corresponding factors are added at the left-hand side of the ITO tree (10).

The ITO formalism for the PES and DMS expansions can be also applied in terms of normal mode coordinates, as described in [32] [31]. This results in a similar expression to eq.(10) where the symmetrized $S R_{j}$ and angular $S A_{k}$ coordinates are replaced by normal coordinates $Q_{t}$. In this work we used the normal mode ITO representation for the dipole moment modeling and for intensity calculations because the matrix elements of the corresponding operators can be computed analytically. However, a sufficiently complete transformation from nonlinear symmetrized coordinates (1-6) to rectilinear normal coordinated $Q_{t}$ in case of $\mathrm{SF}_{6}$ appears to be quite complicated. In this work, we obtained the corresponding relations $\left\{S R_{j}, S A_{k}\right\}$ $\leftrightarrow Q_{t}$ numerically via the solution of the system of non-linear equations. A distribution of the 
RMS deviations of the fit of 15 normal coordinates to 15 internal symmetrized coordinates is shown in Fig 2. The error of the corresponding numerical solution $Q_{t}=Q_{t}\left(S R_{j}, S A_{k}\right)$ was much smaller than the precision of the fit of the analytical form (10) to ab initio points and thus did not affect the accuracy of the final calculation of spectra.

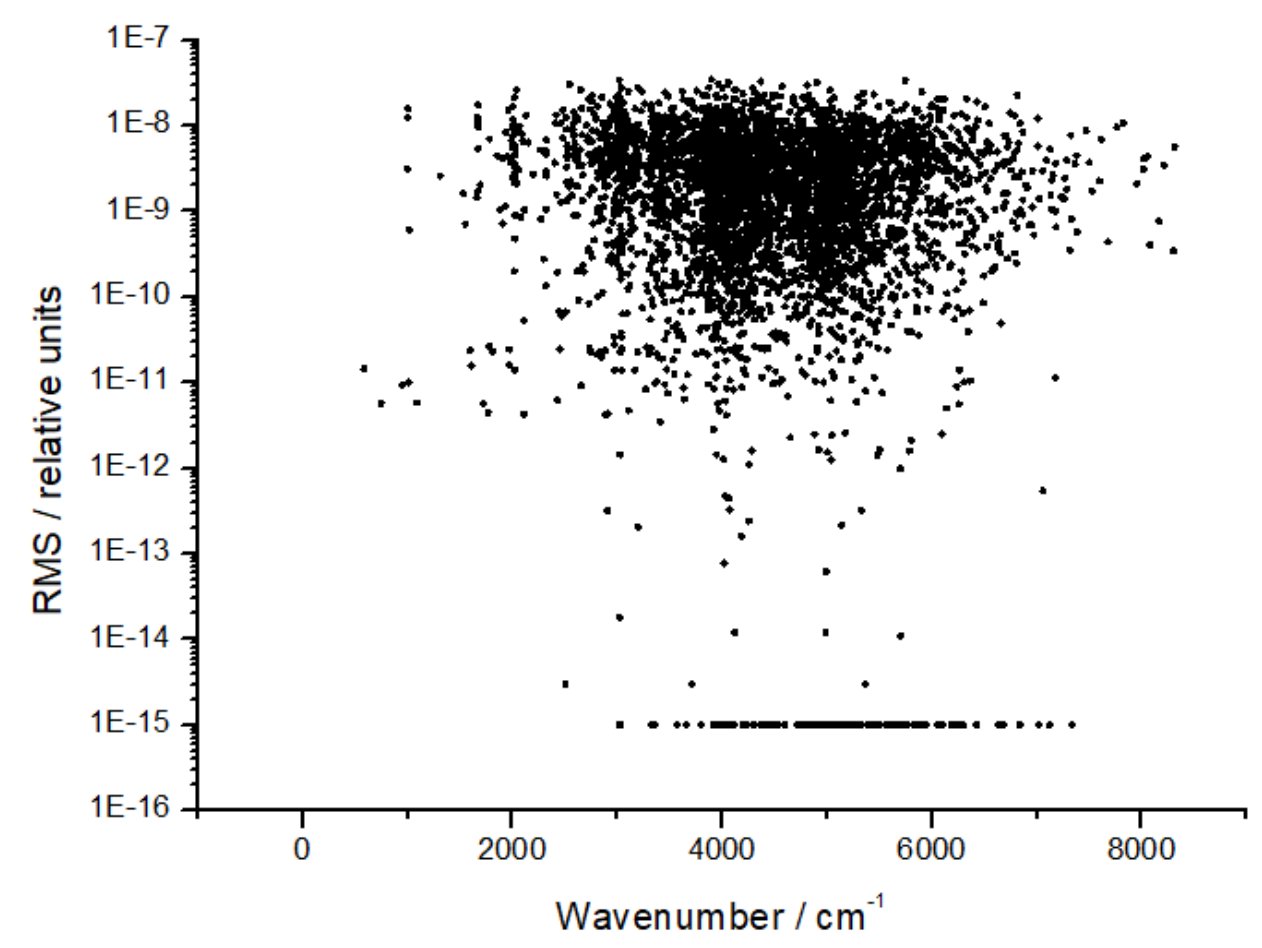

Figure 2. RMS distribution for non-linear fit of normal coordinates $Q_{t}$ to internal symmetrized coordinates $S R_{j}, S A_{k}$

\section{The PES calculation}

To construct the molecular PES of $\mathrm{SF}_{6}$, we first computed a set of ab initio electronic energies at various molecular configurations. Equilibrium geometries and vibrational frequencies were computed with the well-established coupled cluster approach $\operatorname{CCSD}(\mathrm{T})$ with all single and double substitutions from the Hartree-Fock reference determinant augmented by a perturbative treatment of connected triple excitations [53] [54]. It was found to provide a good description for the equilibrium geometry and vibrational frequencies, and then used in this work to calculate the first full-dimensional PES of $\mathrm{SF}_{6}$. We used correlation consistent polarized valence $n$-tuple $\zeta$ basis sets of Dunning, Peterson and co-workers [55] [56] [57] [58] for the calculations and specifically adapted Douglas-Kroll basis for calculations including relativistic effects [59]. Energies for all electronic structure calculations were converged to MOLPRO default value $10^{-10}$ a.u. 
In order to obtain accurate calculations of vibrational and rotational energy levels from the theoretical PES useful for spectroscopic analysis, it is desirable to use high level ab initio methods with sufficiently large basis sets in electronic structure calculations. However, in case of $\mathrm{SF}_{6}$, the full-grid calculations with large basis sets (like those recently employed for methane [21] [30]) are too demanding. In this work, an essential part of electronic energy calculations was performed with the CCSD(T)/VTZ ansatz at 6412 nuclear geometries of the grid optimized for the 6-th order PES. To refine the analytical PES form, the same method and the same basis set was additionally used at 2296 geometries of the 8-th order grid. In order to control the surface shape at larger nuclear displacements we carried out supplementary MP2 calculations with VTZ atomic basis on extended grid of 85136 geometries also optimized for the 8-th order PES.

Empirical PES corrections are often used to compensate the lack of accuracy of ab initio calculations. However, for an efficient application of an empirical optimization these corrections have to be relatively small. Typically, simple empirical corrections are used to reduce calculated minus observed deviation for the fundamental vibration frequencies, but this could produce uncontrolled errors for highly excided states if the shape of the initial ab initio PES is not reliably established. This is an important point for a correct modeling of "hot" bands as they could produce a significant contribution the atmospheric absorbance by heavy molecules with relatively low vibrational modes. In case of $\mathrm{SF}_{6}$, only a limited number of experimental vibrational frequencies is known, that can be used for a validation of $a b$ initio results. Ab initio equilibrium geometry obtained with CVQZ and CVQZ-DK basis sets were close to empirical ones ( Table 1), but calculations using CCSD(T)/ CVQZ-DK ansatz for a large grid of geometries is actually too demanding. For this reason, we have applied the algorithm of corrections similar to that of ref. [49]. First, we have computed the one-dimensional «radial» PES section using the CVQZ-DK basis set as well as electronic energies at 67 nuclear geometries sufficient to determine the force field up to the third order. Then the difference between electronic energies in 67 geometries using CVQZ-DK and VTZ basis sets was fitted to an analytical function $F_{O 3}(S(r, q))$ of 15 symmetrized coordinates expanded up to 3-rd order. One dimensional radial correction functions $f_{X}(r)$ were constructed as a difference between two radial potentials calculated for X-basis and for VTZ basis

$$
f_{X}(r)=V_{X}(r)-V_{V T Z}(r)-F_{O 3}\left(S\left(r, r_{e}, r_{e}, \cdots, q_{e}\right)\right)
$$

where $V_{X}(r)$ stands for electronic energies at the radial cut and $\mathrm{X}=\mathrm{CVQZ}-\mathrm{DK}$ or VQZ. The additional one-dimensional function $F_{O 3}(r, 0,0, \cdots, 0)$ was obtained from the third order correction. All other bond lenghts were fixed to their equilibrium values for $X$ and VTZ basis sets whereas all angles were fixed to their equilibrium values. Functions $f_{X}(r)$ for $X=\mathrm{CVQZ}$ - 
DK is shown in Fig. 3. Note that the one-dimensional radal correction (11) near the equilibrium geometries of VTZ and $X$ basis sets quite significantly depends on the analytical representation.

The number $N$ of irreducible tensors of the total $A_{1 g}$ representation involved in the PES expansion (9-10) increases quite rapidly with the order. Up to the orders $2,3,4,5,6,7$, and 8 this number $\mathrm{N}$ is equal to $9,31,123,406,1334,3924$, and 10994, respectively. For PES parameterization, to provide the best results using the least square technique, more complex onedimensional basis functions are used in (1-6). Morse-type functions are used as primitive radial functions

$$
f_{r}\left(r_{i}\right)=1-\exp \left(-\alpha\left(r_{i}-r_{e}\right)\right)
$$

involved in the definitions of the symmetrized $S R_{\Gamma}$ and angular $S A \Gamma$ coordinates which are used to build our final analytical PES model. Although the function $f_{r}(r)^{2}$ for $\alpha=2.0$ reproduces very accurately the one-dimensional potential of SF, but the best choice of $\alpha$ in (12) for fulldimensional PES was $\alpha=1.9$. Consequently, our PES model tends to the corresponding finite asymptote at large inter-nuclear distances contrary to a simple power series expansion in $\left(r-r_{e}\right)$. The angular function was expressed in terms of the cosine function:

$$
f_{a}\left(q_{i j}\right)=\cos \left(q_{i j}\right)+\alpha \cos ^{2}\left(q_{i j}\right) .
$$

Similar to methane PES [49], it is preferable to use the value $\alpha=1 / 3$ for angular functions.

The relevance of various analytical representation of the PES was checked by the quality of the PES fit on the 8-th order grid of 85136 geometries using MP2/VTZ calculated energies. Various orders of the PES expansion and various forms of the angular dependence were tested. The results of the least-squares fits using weight function $w(E)$ similar to that previously employed for methane studies [60] [49]

$$
w(E)=\frac{\tanh \left(-0.0005\left(E-E_{1}\right)+1.002002002\right)}{2.002002002}
$$

where $E_{1}=7000 \mathrm{~cm}^{-1}$, are collected in Table 3 . It is seen that parameter value $\alpha=1 / 3$ permitted a notable improvement of the standard deviation of the fit for 4-th and 5-th order of the PES expansion. The use of supplementary (redundant) coordinated, as reported in the case of methane [51] [21] could significantly change the PES near the equilibrium. In this work we have also evaluated a possible impact of supplementary coordinates (7), (8) for $\mathrm{SF}_{6}$. An introduction of $S$ ' coordinate in the PES expansion has somewhat larger effect on the standard deviation than that of $S$, but in both cases the improvement of the standard deviation was smaller with respect to the inclusion of $\alpha=1 / 3$ in the quadratic terms of Eq.(13). Finally, we consider that the fifthorder PES expansion provides an acceptable quality of the fit of ab initio energies up to $7000 \mathrm{~cm}^{-}$ 1 . 
Table 3. Result of various PES fits using 85136 electronic MP2/VTZ energies.

\begin{tabular}{|c|c|c|c|c|c|}
\hline Order & $\begin{array}{c}\text { Additional angular } \\
\text { coordinates: order, type }\end{array}$ & \#Parameters & Alpha & $\begin{array}{c}\text { RMS } \\
\mathrm{cm}^{-1}\end{array}$ & $\begin{array}{c}\text { STD } \\
\mathrm{cm}^{-1}\end{array}$ \\
\hline 4 & - & 123 & 0. & 8.29 & 3.71 \\
\hline 4 & O3, S' & 151 & 0. & 6.77 & 3.16 \\
\hline 4 & O3, S” & 151 & 0. & 8.08 & 3.62 \\
\hline 4 & - & 123 & $1 / 3$ & 4.58 & 2.13 \\
\hline 4 & O2, S' & 128 & $1 / 3$ & 3.98 & 1.90 \\
\hline 4 & O2, S" & 128 & $1 / 3$ & 4.27 & 2.05 \\
\hline 4 & O3, S' & 151 & $1 / 3$ & 3.48 & 1.70 \\
\hline 4 & O3, S" & 406 & 0. & 1.96 & 0.79 \\
\hline 5 & - & 434 & 0. & 1.57 & 0.68 \\
\hline 5 & O3, S' & 434 & 0. & 1.66 & 0.68 \\
\hline 5 & O3, S” & 406 & $1 / 3$ & 0.94 & 0.43 \\
\hline 5 & - & 434 & $1 / 3$ & 0.58 & 0.28 \\
\hline 5 & O3, S' & 434 & $1 / 3$ & 0.75 & 0.34 \\
\hline 5 & O3, S” & & & & \\
\hline
\end{tabular}

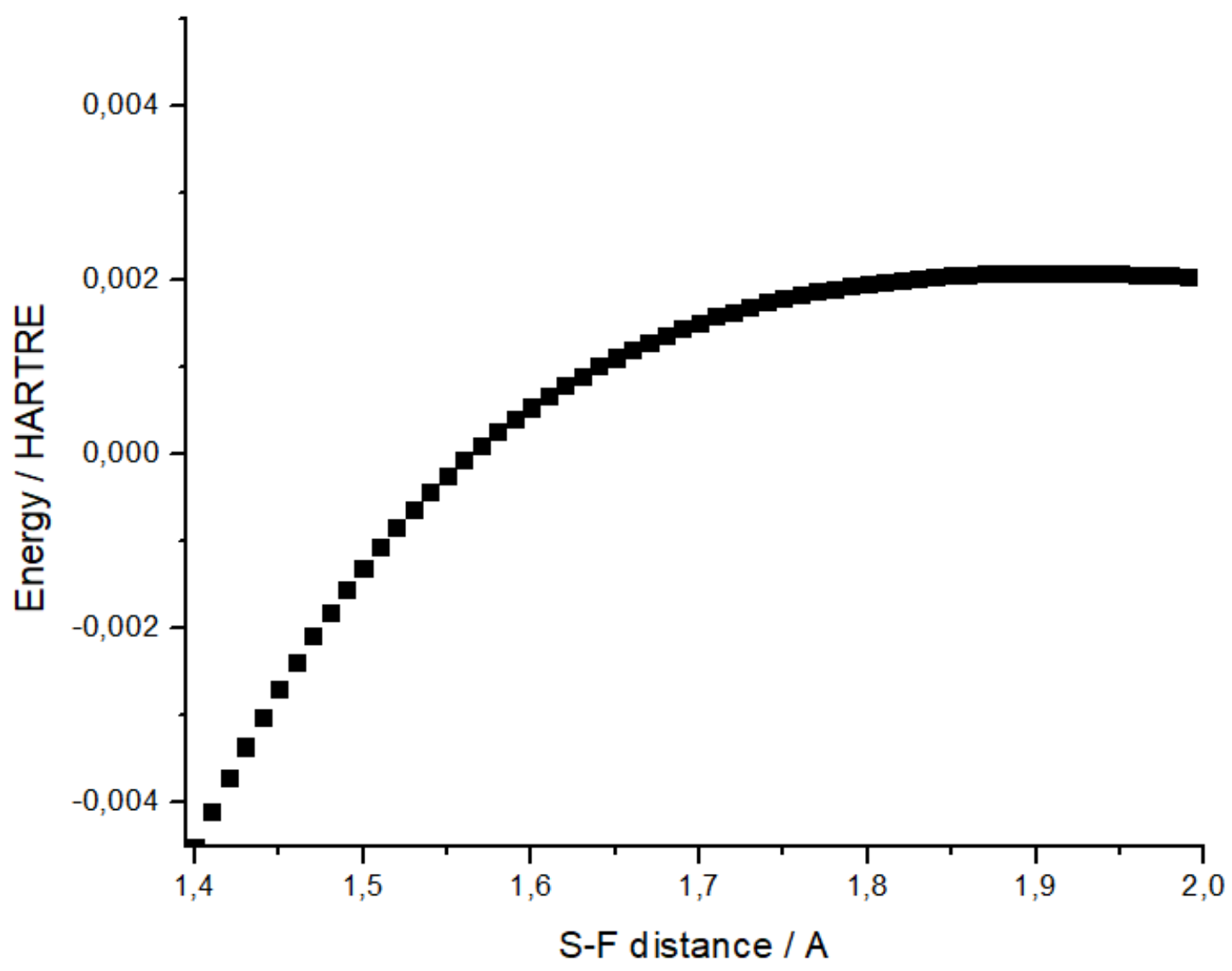


Figure 3 - One-dimensional radial correction E $\{$ CCSD(T)-DK/CVQZ-DK $\}$ $\mathrm{E}\{\mathrm{CCSD}(\mathrm{T}) / \mathrm{VTZ}\}$.

The comparison of the observed fundamental band centers of ${ }^{32} \mathrm{SF}_{6}$ with our variational nuclear motion calculations using ab initio $\operatorname{CCSD}(\mathrm{T}) / \mathrm{VTZ}$ and $\operatorname{CCSD}(\mathrm{T}) / \mathrm{CVQZ}-\mathrm{DK}$ surfaces is given in Table 4. For the VTZ surface defined on a large grid, vibrational and rotational energies were computed using TENSOR variational code in normal mode ITO representation. Technical detail can be found in the previous works devoted to five- and six-atomic molecules [25] [32] [33] [34]. For the VQZ surface defined on a small grid, we used an incremental scheme accounting for the relative difference $\omega_{i}(V Q Z)-\omega_{i}(V T Z)$ between the harmonic frequencies in these basis sets as described below.

The five of six fundamentals calculated with the CVQZ-DK basis set accounting for relativistic contributions show in average better agreement with observed frequencies than VTZ calculations. Note that the most significant contribution in the increase of the fundamental frequencies of angular modes $v_{4}, v_{5}, v_{6}$ when using the CVQZ-DK PES in comparison with the VTZ PES is due to the decrease of the equilibrium S-F bond length. A rough estimation of the deviations between VQZ calculations and the observed fundamental band centers (as given in column 4 of Table 4) was obtained using approximate relation $v_{i}(V Q Z)=v_{i}(V T Z)+\left(\omega_{i}(V Q Z)-\right.$ $\left.\omega_{i}(V T Z)\right)$ where $\omega_{i}$ are harmonic frequencies.

Table4: The fundamental band centers $\left(\mathrm{cm}^{-1}\right)$ for ${ }^{32} \mathrm{SF}_{6}$ : comparison of purely ab initio calculations using the VTZ and CVQZ-DK PESs with experimental values.

\begin{tabular}{|l|l|l|l|l|l|l|}
\hline & Obs & $\begin{array}{l}\text { Calc } \\
\text { PES VTZ } \\
r_{e}=1.57015\end{array}$ & $\begin{array}{l}\text { Obs-Calc } \\
\text { VTZ }\end{array}$ & $\begin{array}{l}\text { Obs-Calc } \\
\text { PES VQZ* } \\
r_{e}=1.57015\end{array}$ & $\begin{array}{l}\text { Calc PES } \\
\text { CVQZ-DK } \\
r_{e}=1.5613\end{array}$ & $\begin{array}{l}\text { Obs-Calc } \\
\text { CVQZ-DK }\end{array}$ \\
\hline$v_{1}$ & 774.5445 & 780.814 & 5.842 & 13.717 & 773.178 & 1.365 \\
\hline$v_{2}$ & 643.3736 & 662.137 & -12.113 & -1.070 & 646.928 & -3.5546 \\
\hline$v_{3}$ & 947.9763 & 982.123 & -25.218 & -5.373 & 958.800 & -10.824 \\
\hline$v_{4}$ & 614.9819 & 613.344 & 5.353 & 11.756 & 620.836 & -5.854 \\
\hline$v_{5}$ & 523.449 & 519.258 & 8.766 & 12.495 & 525.897 & -2.448 \\
\hline$v_{6}$ & 347.985 & 347.329 & 3.606 & 7.479 & 351.724 & -3.739 \\
\hline
\end{tabular}

*These VQZ values in the fourth column were obtained from full dimensional variational nuclear motion calculation at the VTZ level corrected by the difference $\omega_{i}(V Q Z)-\omega_{i}(V T Z)$ between the harmonic frequencies. The values of $r_{e}$ are given in $\AA$.

The S-F bound length computed in CVQZ-DK basis set approached towards the empirical value. This could simplify in future an empirical optimization of the PES parameters. But in order to improve the RMS deviation of the 15-dimensional PES fit with a rather small number of 
parameters, the slightly increased value $r_{e}=1.5613$ (instead of 1.557 for CVQZ-DK) was used. The final $a b$ initio PES of this work was constructed using the least-squares fit of the $F_{p}$ parameters of the 5-th order expansion (9) to CCSD(T)/VTZ electronic energies at 8708 geometrical nuclear configurations with the CCSD(T)/CVQZ-DK correction as described above. In order to improve the fit accuracy near the bottom of the PES the weighting function (13) with the parameter $E_{l}=5000 \mathrm{~cm}^{-1}$ was applied. This permitted obtaining RMS deviation of $0.5 \mathrm{~cm}^{-1}$ for 4283 geometries in the range of $5000 \mathrm{~cm}^{-1}$ above the potential minimum. Since the order of the PES was not very high (in comparison with best methane calculations [21]), the MP2/VTZ electronic energies were not explicitly included in the fit, but were used to check the PES behavior for larger nuclear displacements.

\section{Ab initio DMS fit}

Several studies [8-12] have been devoted to line-by-line analyses of experimental Raman and infrared $\mathrm{SF}_{6}$ spectra using effective Hamiltonian and effective transition moments models. An accurate determination of empirical line intensities of heavy molecules with low frequency modes is an extremely difficult task because of crowded spectra and blended lines. One of the most challenging issue is a separation of contributions of overlapping cold and hot bands with a significant impact on the uncertainty in the temperature dependence of the absorption or emission of radiation.

Advanced $a b$ initio calculations of molecular PESs and DMS together with the development of variational methods for nuclear motion calculations ( [61] [62] [63] [64] [65] [18] [66] and references therein) permitted constructing quite complete lines lists for triatomic [61], [67], [68] [69] [70], four-atomic [17] [31] [71] [72] [73], five-atomic [34] [74] [30] [19] [22] [75] and six-atomic [23] [24] [25] [76] molecules. In some of these cases the accuracy of ab initio line intensities were competitive [77] [78] [79] [80] with best empirical determinations being more complete and reliable for the hot bands [68] [81] [82] [7] [83] [73]. On the other hand, knowledge of the shape of ab initio surfaces [84] [85] helped understanding issues related to molecular dynamics.

One more atom in a molecule produces three supplementary degrees of freedom in the nuclear motion resulting to a very substantial computational effort for $a b$ initio predictions of high-resolution spectra for polyatomic molecules. In a brute-force approach (without symmetry considerations) there would be roughly a factor $\sim N^{6}$ of dimensionality between global spectra calculations of five-atomic molecule ( like methane) and seven-atomic molecule like $\mathrm{SF}_{6}$ where $N$ is a number of ab initio grid points or of basis set function for each degree of freedom. 
Here we present the first results for the full 15-dimensional $\mathrm{SF}_{6} \mathrm{DMS}$ components and for intensity calculations in the range of the strongest bands. A full account of symmetry permitted drastically reducing the number of redundant geometries and the number of independent DMS parameters. For the construction of the DMS we used the corresponding irreducible tensor representation similar to the analytical expansions (9-10) where the symmetrized internal coordinates were replaced by normal mode coordinates. The corresponding procedure has been described in our previous works [86] [71] devoted to four-atomic and five-atomic molecules. In these works, the ab initio DMSs were first built in molecular-fixes frames corresponding to the axes choice options of the MOLPRO code. Then the axes were rotated to the Eckart system. As in case of $\mathrm{SF}_{6}$ the relations between internal and normal coordinated $\mathrm{Q}_{\mathrm{i}}$ were found via numerical transformation, it was easier to proceed by direct DMS fits in normal coordinates.

A progress in methane $a b$ initio calculations has taken a couple of decades $[\mathbf{8 7}][\mathbf{6 0}][\mathbf{8 8}]$ [81] [21]. The line intensity accuracy in infra-red of $1 \%$ to $5 \%$ has been recently reported [80] using the Coupled-Cluster method with ACV5Z one-particle basis sets. In case of $\mathrm{SF}_{6}$ such calculations are presently too demanding. In this work we used $\operatorname{CCSD}(\mathrm{T})$ method with two basis sets for the dipole moment calculations. The calculations using VTZ basis were conducted on the first grid containing 1664 nuclear geometries, which was a sub-set of the 6-th order grid relative to PES computations described above. This was then supplemented by AVTZ calculations at 500 geometries. In both cases the optimal samples of geometries near the bottom of the PES were determined. At each geometry, three projections of the dipole moment were obtained. Dipole moments were computed as the derivative of the energy with respect to the weak external uniform electric field using the finite difference scheme with the field variation of 0.0002 a.u. around at the zero field strength.

For the DMS construction we used here the scheme, which has been employed in our previous works for PES and DMS of four-to-six atomic molecules [71] [86] [22] [24]. After having computed the dipole moments with AVTZ and VTZ basis sets we fitted the corresponding 15-dimensional function diff $2_{-} O_{3}\left(Q_{i}\right)$, which describes the corresponding difference DMS(AVTZ) - DMS(VTZ). This function was modeled by the third-order ITO expansion containing 61 adjustable parameters, of which 50 parameters were statistically welldefined. Then, for supplementary geometries from $N=501$ to $N=1664$ were the AVTZ calculations were lacking, the AVTZ dipole moments were evaluated as VTZ dipole moments plus diff__o3 $\left(Q_{i}\right)$. Finally, the forth-order parameters of the DMS power series expansion in normal coordinated were fitted to the above defined ab initio dipole moment values using weight function (14) with $E_{l}=10000 \mathrm{~cm}^{-1}$. Of 260 fourth-order DMS parameters 194 ones were statistically well-determined. This procedure conducted to high accuracy of the fit corresponding 
to the RMS deviation of $1.4 \mathrm{E}-5$ a.u. and the weighted standard deviation of $9.9^{*} 10^{-6}$. The distribution of the fit discrepancies is shown in Fig. 4.

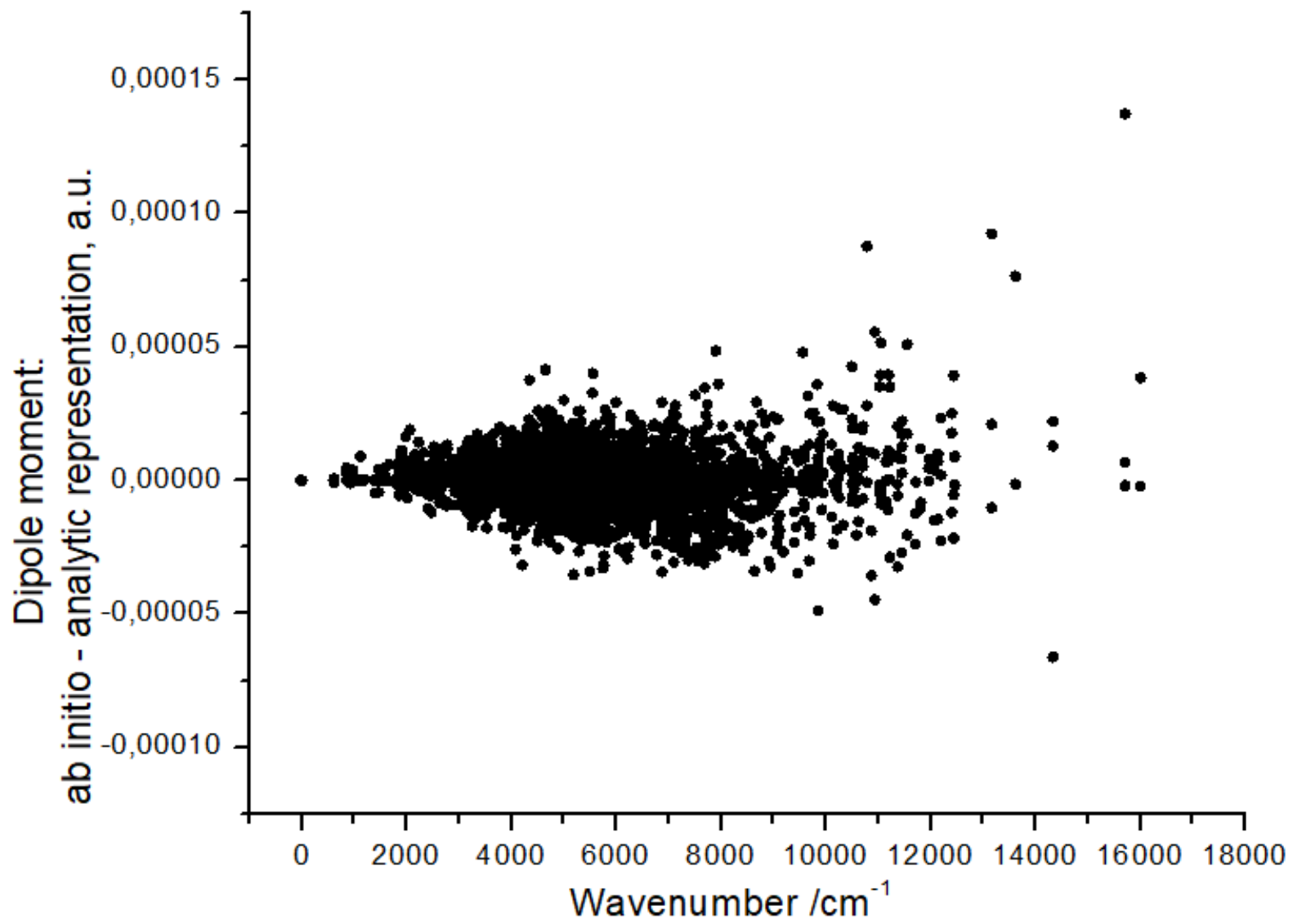

Figure 4 - Deviations between fitted analytical representation of the DMS and ab initio dipole moment values versus energies at the corresponding nuclear geometries.

\section{Results and Conclusion}

Preliminary evaluation of $\mathrm{SF}_{6}$ band intensities using algorithms of variational method described in [31] [32] [33] [34] permits to conclude that our PES and DMS provided realistic predictions of absorption spectra, at least in the range of strong bands. The comparison of our calculated absorption cross-sections with experimental $\mathrm{SF}_{6}$ cross section [89] [13] shows a very good agreement at cold temperature of $180 \mathrm{~K}$, see Fig. 5, lower panel. Note that calculated band centers of ${ }^{32} \mathrm{SF}_{6}$ and ${ }^{34} \mathrm{SF}_{6}$ isotopologues in Fig. 5 were shifted to empirical values $\left(964.196 \mathrm{~cm}^{-1}\right.$ and $932.534 \mathrm{~cm}^{-1}$ correspondingly) available at http://vamdc.icb.cnrs.fr/PHP/SF6.php. An agreement of our calculations with experimental absorption cross-sections of Sharp et al [90] at room temperature is also satisfactory (Fig. 5, upper panel). The latter ones have been recorded at 
$\mathrm{T}=298 \mathrm{~K}$ with $\mathrm{N}_{2}$ pressure of 760 Torr with medium resolution of $0.112 \mathrm{~cm}^{-1}$ and included in HITRAN-on-line cross-section compilation [90] [13] [91]. The absorption feature near $930 \mathrm{~cm}^{-1}$ corresponds to the $\mathrm{v}_{3}$ band of the ${ }^{34} \mathrm{SF}_{6}$ isotopologue at natural abundance. Our ab initio crosssections agree much better with these observations than the simulations using $\mathrm{SF}_{6}$ line-by-line lists constructed from effective models as available in HITRAN_2016 [13], GEISA_2015 [14] and Dijon (SheCaSDa) [92] databases as shown in Fig. 5, upper panel. However, ab initio roomtemperature simulations appear to be slightly less accurate than for $180 \mathrm{~K}$ (Fig. 5, lower panel).

Calculations at room temperature require an account of more significant contributions of hot bands involving higher vibrational states. The preliminary data obtained in this work will be made available through the TheoReTS [66] (http://theorets.univ-reims.fr, http://theorets.tsu.ru) information system. A further improvement could be possibly obtained using lager vibrational basis set that is computationally very demanding. We plan to extend the computation of full line lists in future studies. 


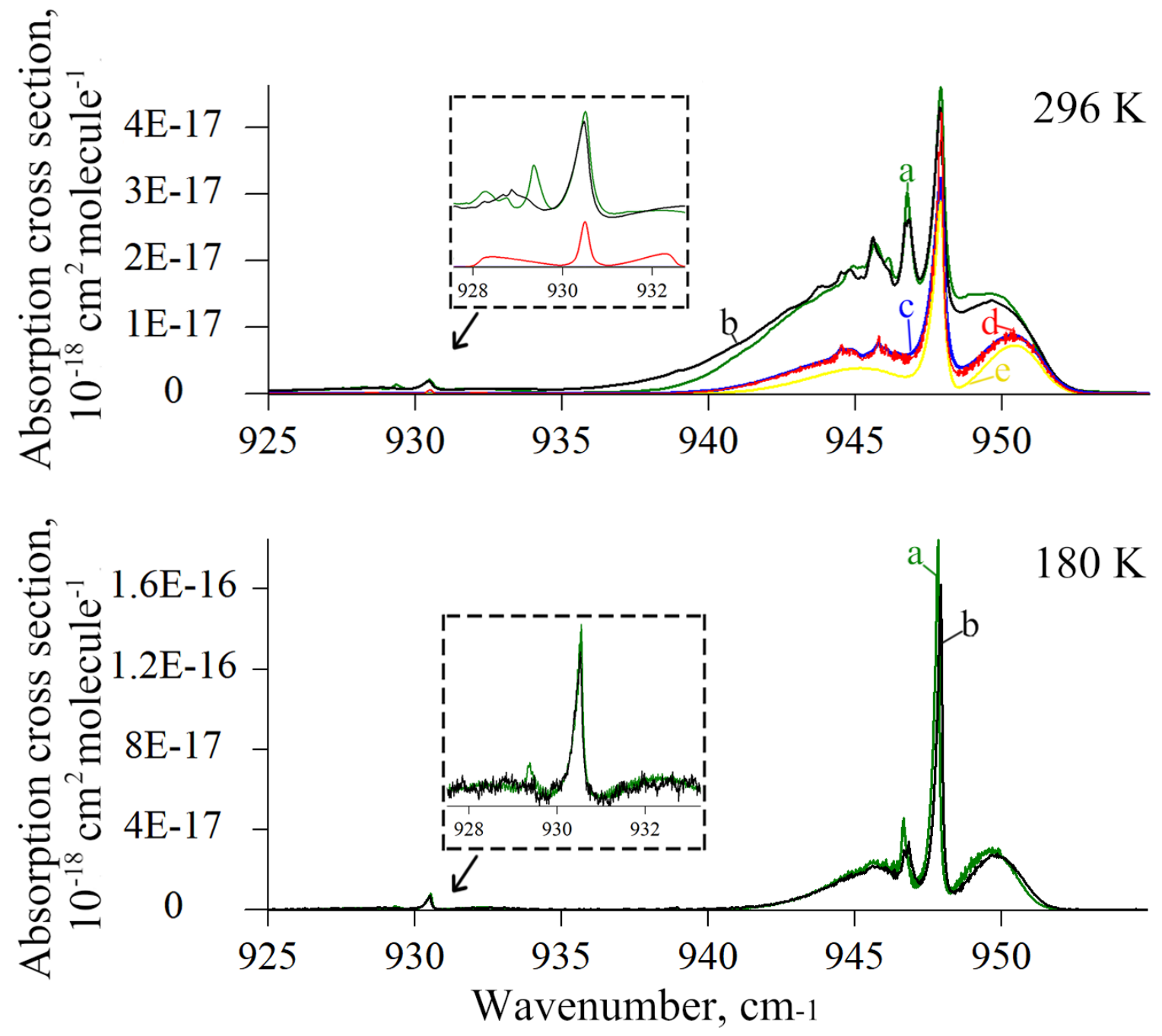

Figure 5. Comparison of cross section at a temperature of 296K (Upper panel) and of 180K (Lower panel). Upper panel: our simulation from line list of TheoReTS (green - a), the experimentally observed PNNL spectrum available in HITRAN (black -b ), simulation from line list of GEISA 2015 (blue -c ), the theoretical calculation of Dijon (red - d) and simulation from line list of HITRAN 2016 database (yellow e). Lower panel: our simulation from line list of TheoReTS (green - a), the experimental observed spectrum from HITRAN (black -b )

\section{Acknowledgements}

The research in IOA was performed as part of the state assignment of the IOA SB RAS.

V.T. acknowledges the support from Academic D..Mendeleev program of Tomsk State University and M.R. form LEFE CHAT CNRS program.

\section{Supplementary Materials}

In the Supplementary Materials, we provide PES in the internal coordinates and DMS in normal mode expansion in an easy to use explicit form for ${ }^{32} \mathrm{SF}_{6}$ 
References

(1) Jesse S. ; Pedraza A.J.; Fowlkes J.D. Etching-enhanced Ablation and the Formation of a Microstructure in Silicon by Laser Irradiation in an $\mathrm{SF}_{6}$ Atmosphere. J. Mater. Res., 2002 , no. 17, 1002.

(2) Johnstone W.M. ; Newell W.R. Absolute elastic differential cross sections for electron scattering from SF6. J. Phys. B: At. Mol. Opt. Phys., 1991, no. 24, $473-487$.

(3) Malik N.H. ; Qureshi A.H. A Review of Electrical Breakdown in Mixtures of SF 6 and Other Gases. IEEE Trans. Electr. Insul., 1979 , 14, no. 1.

(4) Hodnebrog O. ; Etminan M. ; Fuglestvedt J.S. ; Marston G. ; Myhre G. ; Nielsen C.J. ; Shine K.P.; Wallington T.J. Global Warming Potentials and Radiative Efficiencies of Halocarbons and Related Compounds: a Comprehensive Review. Rev. Geophys., 2013 , 51, no. 2, 300 - 378.

(5) Ravishankara A.R. ; Solomon S. ; Turnipseed A.A.; Warren R.F. Atmospheric Lifetimes of Long-Lived Halogenated Species. Science, 1993 , 259, no. 5092, 194-199.

(6) Earth System Research Laboratory Global Monitoring Division. [Online]. http://www.esrl.noaa.gov/gmd/hats/data.html

(7) Rey M ; Chizhmakova I.S. ; Nikitin A.V.; Tyuterev V.G. Understanding global infrared opacity and hot bands of greenhouse molecules with low vibrational modes from first-principles calculations: the case of CF4. Phys. Chem.Chem.Phys., 2018 , 20, 21008--21033.

(8) Boudon V.; Bermejo D. First High-Resolution Raman Spectrum and Analysis of the V5. J. Mol. Spectrosc., 2002 , 213, $139-144$.

(9) Boudon V. ; Pierre G.; Burger H. High-Resolution Spectroscopy and Analysis of the $\mathrm{V}_{4}$ Bending Region of $\mathrm{SF}_{6}$ near $615 \mathrm{~cm}^{-1}$. J. Mol. Spectrosc., 2001 , 205, 304 - 311.

(10) Faye M. ; Boudon V. ; Loëte M. ; Roy P.; Manceron L. Observation and analysis of the $S_{6} V_{2}+v_{4}+v_{5}$ band: Improved parameters for the $v_{5}=1$ state. J. Molec. Spectrosc., 2016 , 325, 35-41.

(11) Faye M. ; Boudon V. ; Loëte M. ; Roy P.; Manceron L. The high overtone and combination levels of $\mathrm{SF}_{6}$ revisited at Doppler-limited resolution: A global effective rovibrational model for highly excited vibrational states. J. Quant. Spectrosc. and Radiat. Transfer., 2017 , 190, 38-47.

(12) Faye M. ; Manceron L. ; Roy P. ; Boudon V.; Loëte M. First analysis of the $v_{3}+v_{5}$ combination band of $\mathrm{SF}_{6}$ observed at Doppler-limited resolution and effective model for the $\mathrm{v}_{3}+\mathrm{v}_{5}-\mathrm{v}_{5}$ hot band. J. Molec. Spectrosc., 2018 , 348, 37-42.

(13) Gordon I.E. ; Rothman L.S. ; Hill C. ; Kochanov R.V. ; Tan Y. ; Bernath P.F.; al. et The HITRAN2016 molecular spectroscopic database. J Quant Spectrosc Radiat Transfer, 2017 , 203, 3-69.

(14) Jacquinet N. ; al. et The 2015 edition of the GEISA spectroscopic database. J. Molec. Spectrosc., 
$2016,327,31-72$.

(15) Yurchenko S. ; Carvajal M. ; Thiel W.; Jensen P. Ab initio dipole moment and theoretical rovibrational intensities in the electronic ground state of $\mathrm{PH}_{3}$. J. Molec. Spectrosc., 2006 , 239, 7187.

(16) Nikitin A.V. ; Holka F. ; Tyuterev VI.G.; Fremont J. Vibration energy levels of the $\mathrm{PH}_{3}, \mathrm{PH}_{2} \mathrm{D}$, and $\mathrm{PHD}_{2}$ molecules calculated from high order potential energy surface. J. Chem. Phys, 2009 , 131, 244312.

(17) Huang X. ; Schwenke D.W.; Lee T.J. Rovibrational spectra of ammonia. II. Detailed analysis, comparison, and prediction of spectroscopic assignments for ${ }^{14} \mathrm{NH}_{3},{ }^{15} \mathrm{NH}_{3}$, and ${ }^{14} \mathrm{ND}_{3}$. J. Chem. Phys., 2011 , 134, 044321.

(18) Yurchenko S.N. ; Tennyson J. ; R.J. Barber; Thiel W. Vibrational transition moments of $\mathrm{CH}_{4}$ from first principles. J. Mol. Spectrosc., 2013 , 291, 69-76.

(19) Owens A. ; Yurchenko S.N. ; Yachmenev A. ; Tennyson J.; Thiel W. Accurate ab initio vibrational energies of methyl chloride. J. Chem. Phys., 2015 , 142, 244306.

(20) Owens A. ; Yurchenko S.N. ; Yachmenev A.; Thiel W. A global potential energy surface and dipole moment surface for silane. J. Chem. Phys., $2015,143,244317$.

(21) Nikitin A.V. ; Rey M.; Tyuterev VI.G. First fully ab initio potential energy surface of methane with a spectroscopic accuracy. J. Chem. Phys., 2016 , 145, 114309.

(22) Nikitin A.V. ; Rey M. ; Rodina A. ; Krishna B.M.; Tyuterev VI.G. Full-Dimensional Potential Energy and Dipole Moment Surfaces of $\mathrm{GeH}_{4}$ Molecule and Accurate First-Principle Rotationally Resolved Intensity Predictions in the Infrared. J. Chem. Phys. A, 2016, 120, 8983-8997.

(23) Delahaye T. ; Nikitin A. ; Rey M. ; Szalay P.; Tyuterev VI.G. A new accurate ground-state potential energy surface of ethylene and predictions for rotational and vibrational energy levels. J. Chem. Phys., $2014,141,104301$.

(24) Delahaye T. ; Nikitin A.V. ; Rey M. ; Szalay P.G.; Tyuterev V.G. Accurate 12D dipole moment surfaces of ethylene. Chem. Phys. Letters, 2015 , 639, 275-282.

(25) Rey M. ; Delahaye T. ; Nikitin A.V.; Tyuterev V.G. First theoretical global line lists of ethylene $\left({ }^{12} \mathrm{C}_{2} \mathrm{H}_{4}\right)$ spectra at various temperatures in the far-infrared range for quantification of absorption and emission in planetary atmospheres. Astronomy and Astrphysics, 2016 , DOI: 10.1051/0004-6361.

(26) Manzhos S ; Carrington T ; Laverdure L; Mosey N Computing the Anharmonic Vibrational Spectrum of $\mathrm{UF}_{6}$ in 15 Dimensions with an Optimized Basis Set and Rectangular Collocation. J. Phys. Chem. A, $2015,119,9557-9567$.

(27) Burcl R. ; Handy N.C.; Carter S. Vibrational spectra of furan, pyrrole, and thiophene from a density functional theory anharmonic force field. Spectrochimica Acta - Part A, 2003 , 59, 1881-1893.

(28) Wang X. ; Huang X. ; Bowman J.M.; Lee T.J. Anharmonic rovibrational calculations of singlet cyclic $\mathrm{C}_{4}$ 
using a new ab initio potential and a quartic force field. Journal of Chemical Physics, 2013 , 139, 224302.

(29) Pietropolli Charmet A. ; Bizzocchi L. ; Giuliano B.M.'Caselli, P. ; Craig N.C.; Krasnoshchekov S.V. Disentangling the IR spectra of 2,3,3,3-tetrafluoropropene using an ab initio description of vibrational polyads by means of canonical Van Vleck perturbation theory . 2333. Journal of Quantitative Spectroscopy and Radiative Transfer, 2019 , 239, 106656.

(30) Owens A. ; Yurchenko S.N. ; Yachmenev A. ; Tennyson J.; Thiel W. A highly accurate ab initio potential energy surface for methane. J. Chem. Phys., 2016, 145, 104305.

(31) Rey M. ; Nikitin A.V.; Tyuterev VI.G. Ab initio ro-vibrational Hamiltonian in irreducible tensor formalism: a method for computing energy levels from potential energy surfaces for symmetric-top molecules. Molec. Phys., 2010 , 108, 2121-2135.

(32) Rey M. ; Nikitin A.V.; Tyuterev VI.G. Complete nuclear motion Hamiltonian in the irreducible normal mode tensor operator formalism for the methane molecule. J. Chem. Phys., 2012 , 136, 244106.

(33) Rey M. ; Nikitin A.V.; Tyuterev VI.G. First principles intensity calculations of the methane rovibrational spectra in the infrared up to $9300 \mathrm{~cm}^{-1}$. Phys. Chem. Chem. Phys., 2013, 15, no. 25 , 10049-10061.

(34) Rey M. ; Nikitin A.V.; Tyuterev VI.G. Convergence of normal mode variational calculations of methane spectra: Theoretical line list in the icosad range computed from potential energy and dipole moment surfaces. J. Quant. Spectrosc. Radiat. Tranfer., 2015 , 164, 207-220.

(35) Nagarajan G. ; Brinkley D.C. Statistical Thermodynamics Enthalpy, Free Energy, Entropy, and Heat Capacity of Some Hexafluorides of Octahedral Symmetry. Z. Naturforsch., 1971 , 1658-1665.

(36) Spiridonov V.P. ; Tarasov Yu.I. ; Novosadov B.K. ; Nikitin O.Yu.; Maslov I.V. A practical method for diffraction analysis of equilibrium geometries molecules without refined force fields. J. Mol. Struct., $1997,463-470$.

(37) Werner H.-J. ; Knowles P.J. ; Knizia G. ; Manby F.R.; al. et Molpro: a general purpose quantum chemistry program package. WIREs Comput Mol Sci, 2012 , 2, 242--253.

(38) Werner H.- J. ; Knowles P.J ; Lindh R.; al. et. MOLPRO, version 2019.1, a package of ab initio programs; http://www.molpro.net.

(39) Eisfeld W. Highly accurate determination of the electron affinity of $\mathrm{SF}_{6}$ and analysis of structure and photodetachment spectrum of SF 6 . J. Chem. Phys., 2011 , no. 134, 054303.

(40) Miller B.R. ; Fink M. Mean amplitudes of vibration of $\mathrm{SF}_{6}$ and intramolecular multiple scattering. J. Chem. Phys., 1981 , no. 75, 5326.

(41) Champion J.-P. ; Loete M.; Pierre G., Spectroscopy of the Earth's Atmosphere and Interstellar Medium in: K.N. Rao, A.Weber (Eds.). San Diego: Academic Press, 1992. 
(42) Zhilinskii B.I. ; Perevalov V.I.; Tyuterev VI.G., Method of Irreducible Tensorial Operators in the Theory of Molecular Spectra. Novosibirsk: Nauka, 1987.

(43) Chizhmakova I.S. ; Nikitin A.V. Potential Energy Surface of $\mathrm{SF}_{6}$. Atmospheric and Oceanic Optics, 2019,32 , no. 6, 613-618.

(44) Schatz P.N. ; Hornig D.F. Bond Moments and Derivatives in $\mathrm{CF}_{4}, \mathrm{SiF}_{4}$, and $\mathrm{SF}_{6}$ from Infrared Intensities. J. Chem. Phys., 1953 , 21, no. 9, 1516.

(45) Fernandez-Gomez M. ; Lopez-Gonzalez J.J. Calculation of internal valence force constants for $\mathrm{XY}_{6}\left(\mathrm{O}_{h}\right)$ Octahedral molecules. J. Mol. Struct., 1990, 220, 287 - 300.

(46) Pistorius T.C. W. F. Potential Field and Force Constants of Octahedral Molecules. J. Chem. Phys., 1958,29, no. $6,1328-1332$.

(47) Halonen L. ; Child M.S. A local mode model for tetrahedral molecules. Mol. Phys., 1982 , 46, 239255.

(48) Delibas A. ; Aykan V. ; Turkkan D; Akkus H. Point groups in solid state physics I: point group $\mathrm{O}_{\mathrm{h}}$. American Journal of Modern Physics, 2013 , 2, 81-87.

(49) Nikitin A.V. ; Rey M.; Tyuterev VI.G. Rotational and vibrational energy levels of methane calculated from a new potential energy surface. Chem. Phys. Lett., 2011 , 501, 179-186.

(50) Nikitin A.V. Calculation of vibrational energy levels of symmetric molecules from potential energy. Atmospheric and Oceanic Optics, 2015 , 28, no. 5, 379-390.

(51) Nikitin A.V. ; Rey M.; Tyuterev VI.G. An efficient method for energy levels calculation using full symmetry and exact kinetic energy operator: tetrahedral molecules. J. Chem. Phys., 2015 , 142, 094118.

(52) Nikitin A.V. ; Champion J.P.; Tyuterev VI.G. Improved algorithms for the modeling of vibrational polyads of polyatomic molecules: application to $T_{d}, O_{h}$, and $C_{3 v}$ molecules. J. Molec. Spectrosc., 1997 , 182, 72-84.

(53) Raghavachari K. ; Trucks G.W. ; Pople J.A.; Head-Gordon M. Chem. Phys. Lett., 1989 , 157, 479.

(54) Purvis G.D. ; Bartlett R.J. A full coupled-cluster singles and doubles model: The inclusion of disconnected triples. J. Chem. Phys., 1982 , 76, 1910.

(55) Woon D.E. ; Dunning T.H. Gaussian basis sets for use in correlated molecular calculations. III. The atoms aluminum through argon. J. Chem. Phys., 1993 , 98, 1358.

(56) Dunning T.H. Gaussian basis sets for use in correlated molecular calculations. I. The atoms boron through neon and hydrogen. J. Chem. Phys., 1989 , 90, 1007.

(57) Wilson A.K. ; Woon D.E. ; Peterson K.A.; Dunning Jr T.H. Gaussian basis sets for use in correlated molecular calculations. IX. The atoms gallium through krypton. J. Chem. Phys., 1999, 110, 7667. 
(58) DeYonker N.J. ; Peterson K.A.; Wilson A.K. Systematically Convergent Correlation Consistent Basis Sets for Molecular Core-Valence Correlation Effects: The Third-Row Atoms Gallium through Krypton. J. Phys. Chem. A, 2007 , 111 , no. 44, 11383-11393.

(59) de Jong W.A. ; Harrison R.J.; Dixon D.A. Parallel Douglas-Kroll energy and gradients in NWChem: Estimating scalar relativistic effects using Douglas-Kroll contracted basis sets. J. Chem. Phys., 2001 , $114,48$.

(60) Schwenke D.W. ; Partridge H. Vibrational energy levels for $\mathrm{CH}_{4}$ from an ab initio potential. Spectrochim. Acta A, 2001, 57, 887.

(61) Partridge H. ; Schwenke D.W. The determination of an accurate isotope dependent potential energy surface for water from extensive ab initio calculations and experimental data. The Journal of Chemical Physics, $1997,106,4618-4639$.

(62) Marquardt R. ; Quack M. Global Analytical Potential Hypersurface for Large Amplitude Nuclear Motion and Reactions in Methane II. Characteristic Properties of the Potential and Comparison to Other Potentials and Experimental Information. J. Phys. Chem., 2004 , 108, 3166-3181.

(63) Huang X. ; Freedman R.S. ; Tashkun S.A. ; Schwenke D.W.; Lee T.J. Semiempirical ${ }^{12} \mathrm{C}_{16} \mathrm{O}_{2}$ IR line lists for simulations up to $1500 \mathrm{~K}$ and $20000 \mathrm{~cm}^{-1}$.. J.Quant.Spectrosc. Radiat.Transf., $2013,139,134-46$.

(64) Carter S. ; Sharma A.R.; Bowman J.M. First-principles calculations of rovibrational energies, dipole transition intensities and partition function for ethylene using MULTIMODE. Journal of Chemical Physics, 2012 , 137, no. 15, 154301.

(65) Tennyson J. ; Yurchenko S.N. et al. The ExoMol database: molecular line lists for exoplanet and other hot atmospheres. J. Molec. Spectrosc., 2016 , 327, 73-94.

(66) Rey M. ; Nikitin A.V. ; Babikov Y.; Tyuterev VI.G. TheoReTS - An information system for theoretical spectra based on variational predictions from molecular potential energy and dipole moment surfaces. J. Molec. Spectrosc., 2016 , 327, 138-158.

(67) Polyansky O. ; Ovsyannikov R. ; Kyuberis A. ; Lodi L. ; Tennyson J.; Zobov N. Calculation of rotationvibration energy levels of the water molecule with near-experimental accuracy based on an ab initio potential energy surface. J. Phys. Chem. A, 2013 , 117, no. 39, 9633-9643.

(68) Polyansky O. ; Kyberis A. ; Zobov N. ; Tennyson J. ; Yurchenko S.; Lodi L. ExoMol molecular line lists XXX: a complete high-accuracy line list for water. MNRAS, 2018 , 480, no. 2, 2597-2608.

(69) Tyuterev VI.G. ; Kochanov R.V. ; Tashkun S.A. ; Holka F.; Szalay P.G. New analytical model for the ozone electronic ground state potential surface and accurate ab initio vibrational predictions at high energy range. J. Chem. Phys., 2013 , 139, 134307.

(70) Tyuterev V.G. ; Barbe A. ; Jacquemart D. ; Janssen C. ; Mikhailenko S.N.; Starikova E.N. Ab initio predictions and laboratory validation for consistent ozone intensities in the MW, 10 and $5 \mu \mathrm{m}$ ranges. J. Chem. Phys., 2019 , 150, no. 18, 184303. 
(71) Nikitin A.V. ; Rey M.; Tyuterev VI.G High order dipole moment surfaces of $\mathrm{PH}_{3}$ and ab initio intensity predictions in the Octad range. J. Molec. Spectrosc, 2014 , 305, 40-47.

(72) Yurchenko S.N. ; Barber R.J. ; Yachmenev A. ; Thiel W. ; Jensen P.; Tennyson J. A Variationally Computed $\mathrm{T}=300 \mathrm{~K}$ Line List for $\mathrm{NH}_{3}$. J. Phys. Chem. A, 2009 , 113, no. 43, 11845-11855.

(73) Egorov O. ; Nikitin A. ; Rey M ; Rodina A. ; Tashkun S.; Tyuterev VI.G. Global modeling of $\mathrm{NF}_{3}$ line positions and intensities from far to mid-infrared up to $2200 \mathrm{~cm}^{-1}$. JQSRT, $2019,239,106668$.

(74) Rey M. ; Nikitin A.V.; Tyuterev VI.G. Accurate first-principles calculations for ${ }^{12} \mathrm{CH}_{3} \mathrm{D}$ infrared spectra from isotopic and symmetry transformations. J. Chem. Phys., 2014 , 141, 044316.

(75) Nikitin A.V. ; Rey M.; Tyuterev VI.G. Rotational and vibrational energy levels of methyl fluoride calculated from a new potential energy surface. J. Molec. Spectrosc. , 2012 , 274, 28-34.

(76) Mant B.P. ; Yachmenev A. ; Tennyson J.; Yurchenko S.N. ExoMol molecular line lists - XXVII. Spectra of $\mathrm{C}_{2} \mathrm{H}_{4}$. MNRAS, August 2018, 478, no. 9, 3220-3232.

(77) Birk M. ; Wagner G. ; Loos J. ; Lodi L. ; Polyansky O. ; Kyuberis A. ; Zobov N.; Tennyson J. Accurate line intensities for water transitions in the infrared: Comparison of theory and experiment. J. Quant. Spectrosc. Radiat. Transfer, December 2017 , 203, 88-102.

(78) Zak E. ; Tennyson J. ; Polyansky O. ; Lodi L. ; Zobov N. ; Tashkun S.; Perevalov V. Room temperature line lists for $\mathrm{CO}_{2}$ symmetric isotopologues with ab initio computed intensities. J. Quant. Spectrosc. Radiat. Transfer, March 2017 , 189, 267-280.

(79) Tyuterev V.G. ; Kochanov R.V.; Tashkun S.A. Accurate ab initio dipole moment surfaces of ozone: First principle intensity predictions for rotationally resolved spectra in a large range of overtone and combination bands. J. Chem. Phys., 2017 , 146, no. 6, 064304.

(80) Nikitin A.V. ; Rey M.; Tyuterev VI.G. Accurate line intensities of methane from first-principles calculations. J. Quant. Spectrosc. Radiat. Transfer, 2017 , 200, 90-99.

(81) Yurchenko S.N. ; Tennyson J. ExoMol line lists-IV. The rotation-vibration spectrum of methane up to 1500 K. Monthly Notices of the Royal Astronomical Society, 2014 , 440, 1649-1661.

(82) Rey M. ; Nikitin A.V.; Tyuterev VI.G. THEORETICAL HOT METHANE LINE LISTS UP TO T $=2000 \mathrm{~K}$ FOR ASTROPHYSICAL APPLICATIONS. The Astrophysical Journal, 2014 , 788, no. 2, 1.

(83) Rey M. ; Nikitin A.V.; Tyuterev V.G. Accurate Theoretical Methane Line Lists in the Infrared up to $3000 \mathrm{~K}$ and Quasi-continuum Absorption/Emission Modeling for Astrophysical Applications. Astrophysical journal, 2017 , 847, 105.

(84) Tyuterev VI.G. ; Kochanov R.V. ; Campargue A. ; Kassi S. ; Mondelain D.; Barbe A. Does the "reef structure" at the ozone transition state towards the dissociation exist? New insight from calculations and ultrasensitive spectroscopy experiments. Phys.Rev.Lett, 2014 , 113, 143002.

(85) Yuen C.H. ; Lapierre D. ; Gatti F. ; Kokoouline V.; Tyuterev V.G. The Role of Ozone Vibrational 
Resonances in the Isotope Exchange Reaction ${ }^{16} \mathrm{O}{ }^{16} \mathrm{O}+{ }^{18} \mathrm{O} \rightarrow{ }^{18} \mathrm{O}{ }^{16} \mathrm{O}+{ }^{16} \mathrm{O}$ : The Time-Dependent Picture. J. Chem. Phys., 2019 , 123, no. 36, 7733-7743.

(86) Nikitin A.V. ; Rey M.; Tyuterev V.G. New dipole moment surfaces of methane. Chem. Phys.Lett., 2013,565 , no. 5, 5-11.

(87) Lee T.J. ; Martin J.M.L.; Taylor P.R. An accurate ab initio quartic force field and vibrational frequencies for $\mathrm{CH}_{4}$ and isotopomers. J. Chem. Phys., $1995,102,254-261$.

(88) Cassam-Chenai P. ; Lievin J Ab initio calculation of the rotational spectrum of methane vibrational ground state. J. Chem. Phys., 2012 , 136, 174309.

(89) Varanasi. P. (2020, 23.03) HITRAN (Sulfir-containing specis, $\left.\mathrm{SF}_{6}\right)$. [Online]. https://hitran.org/xsc/

(90) Sharpe S.W. ; Johnson T.J. ; Sams R.L. ; Chu P.M. ; Rhoderick G.C.; Johnson P.A. Gas-Phase Databases for Quantitative Infrared Spectroscopy. Appl. Spectrosc., 2004 , 58, 1452-1461.

(91) Johnson T. ; Sams R.; Sharpe S. The PNNL Quantitative Infrared Database for Gas-Phase Sensing: A spectral Library for Environmental, Hazmat, and Public Safety Standoff Detection. Proceedings of SPIE - The International Society for Optical Engineering, 2004 , 5269, 159-169.

(92) Calculated SHeCaSDa line list extraction at 296K. [Online]. http://vamdc.icb.cnrs.fr/PHP/shecasda.php

TOC Graphic

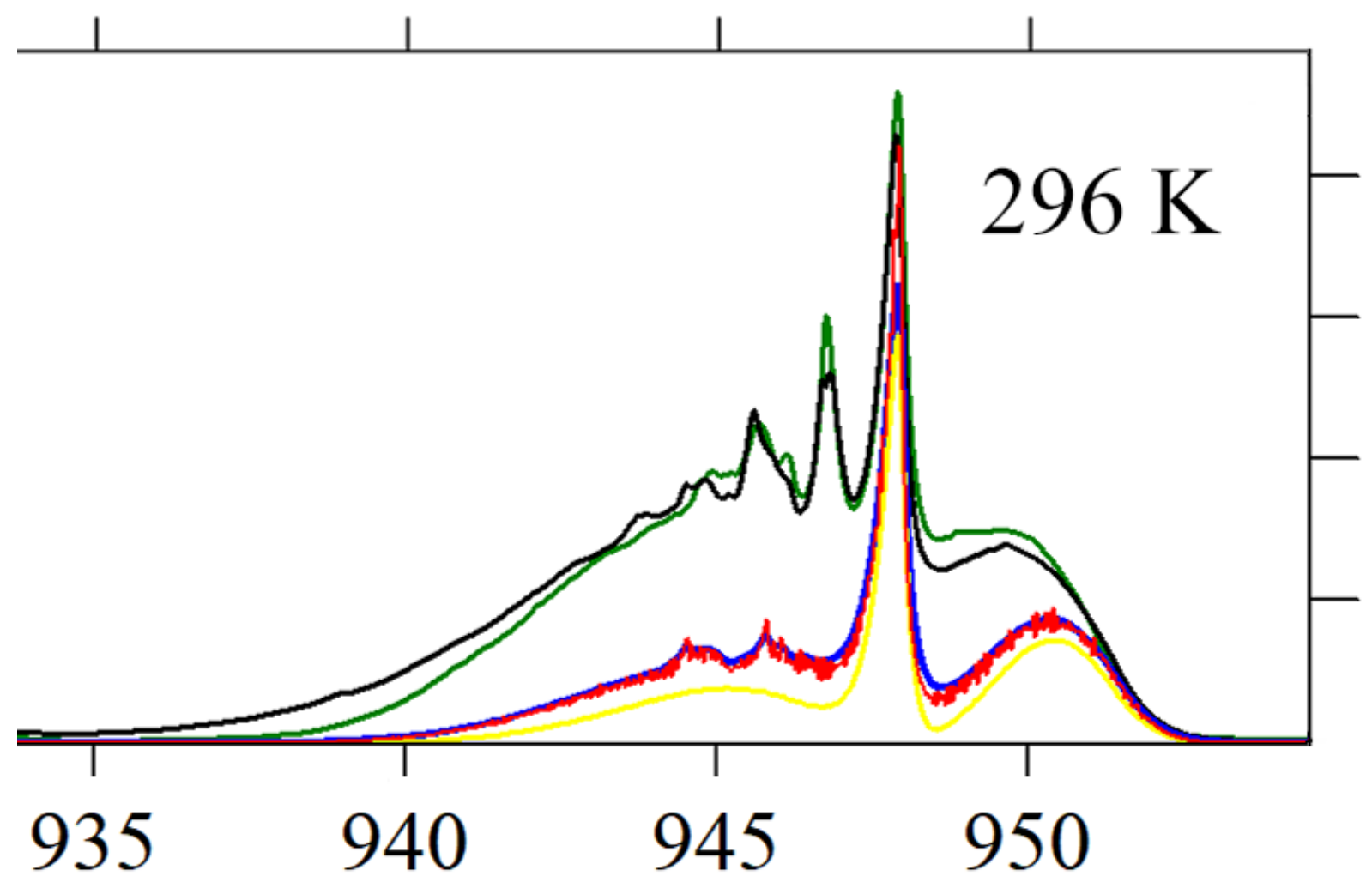

